\title{
LDL as a Cause of Atherosclerosis
}

\author{
Gerald H. Tomkin* and Daphne Owens
}

\author{
Beacon Hospital and Trinity College Dublin 2, Ireland
}

\begin{abstract}
The low density lipoprotein particle is the major transporter of cholesterol around the body and has been shown to be a strong independent risk factor for atherosclerotic events. This review discusses the normal and abnormal metabolism of low density lipoprotein (LDL). Mechanisms by which LDL causes atherosclerosis are discussed with particular reference to the importance of alteration in LDL composition including attachment of other proteins to the circulating LDL particle.
\end{abstract}

Keywords: Low density lipoprotein (LDL), Oxidised LDL, Atherosclerosis, Cholesterol, Proprotein convertase subtilisin kexin type 9 (PCSK9) Niemann Pick C1L1, Apolipoprotein B.

\section{INTRODUCTION}

There has been a huge increase in our understanding of the atherosclerotic process but there also has been a huge increase in both patients with the condition and those who potentially will in the future have the condition. The rising tide of obesity, diabetes and hypertension together with our reluctance to exercise and desire for fast unhealthy food, has made atherosclerosis of major importance economically, politically, scientifically and medically. Treatment of the condition with procedures such as by pass operations, insertion of stents and pharmaceutical agents to treat hypertension, diabetes and dyslipidaemia, pose an enormous burden on society.

The atherosclerotic plaque is cholesterol and fatty acid laden and research efforts have been successful in unraveling some of the mechanisms involved in the formation and rupture of these plaques. The lipoproteins are the major transporters of both cholesterol and fatty acids. Low density lipoproteins (LDL) is the best known lipoprotein not only because Brown and Goldstein [1] discovered the receptor for LDL and demonstrated the importance of the receptor in maintaining cholesterol homeostasis but also because statins, which inhibit 3-hydroxy-3-methylglutaryl coenzyme A (HMG-CoA) reductase, the rate limiting enzyme for cholesterol synthesis, were shown to up regulate the LDL receptor, lower cholesterol in the blood stream and reduce cardiovascular events by about $30 \%$ [2]. Indeed the popularity of the statin drugs has been such that it has been proposed that they should be given to every one in middle age. However, this is probably not cost effective [3].

\section{CHOLESTEROL ABSORPTION AND TRANSPORT}

Cholesterol may be synthesised, the majority in the liver and about $25 \%$ in the intestine, may be absorbed from the diet or reabsorbed from bile. Cholesterol from the intestine is delivered to the liver by the intestinally-derived, apolipoprotein apo B 48 containing lipoprotein the chylomicron, a

\footnotetext{
*Address correspondence to this author at the Diabetes Institute of Ireland, Beacon Clinic, Sandyford, Dublin 18, Ireland; Tel: 0035386 6055031; E-mail: gerald.tomkin@tcd.ie
}

large lipid rich particle with a short half life of circa $90 \mathrm{~min}$. The chylomicron acquires apo E, another apolipoprotein which is transferred to the chylomicron from high density lipoprotein (HDL) in the circulation, and is partially delipidated by lipoprotein lipase before being taken up by the $\mathrm{B} / \mathrm{E}$ receptor in the liver [1]. The cholesterol released is repackaged, together with de novo synthesized and hepatically derived, cholesterol, triglyceride and phospholipids, solubilised by apo B 100 and excreted as very low density lipoprotein (VLDL) into the circulation. The VLDL particles are delipidated by lipoprotein lipase attached to the capillary wall which removes most of the triglyceride and the particle becomes a VLDL remnant. This may be further degraded to form LDL. Apo $\mathrm{E}$ is involved in clearance of most of the delipidated VLDL via the LDL B/E receptor in the liver. Apo $\mathrm{E}$ is then transferred back to HDL. Under normal circumstances LDL catabolism depends on the particle uptake by the LDL receptor which is present in almost all the cells of the body [1]. Thus, LDL is a vehicle to supply cholesterol all over the body in order to maintain cell viability and to provide cholesterol for the synthesis of the steroid hormones. HDL plays a part in reverse cholesterol transport and also protects LDL from oxidation [4] (Fig. 1).

The regulation of LDL is finely tuned. Most cells have the ability to synthesise cholesterol through the HMGCoA reductase pathway, a safety net when cholesterol absorption is diminished. This is well demonstrated by the effect of the statins which inhibit HMGCoA reductase. Reduction in endogenous cholesterol synthesis up-regulates the LDL receptor and stimulates LDL clearance [5]. However it also stimulates cellular cholesterol absorption from the intestine [6]. The mechanism by which cholesterol absorption is regulated was discovered when it was found that ezetimibe, a compound that was known to reduce serum cholesterol, has been shown to bind to the brush borders of the enterocyte and to NPC1L1 and reduce cholesterol transport [7]. Cholesterol absorption is further regulated by ATP binding cassette proteins (ABC) G5 and G8 in the intestine [8]. These proteins work in tandem to re-excrete plant sterols virtually completely and cholesterol to a lesser extent [8]. The genes were also found to be expressed in the liver where 


\section{Cholesterol absorption and LDL formation}

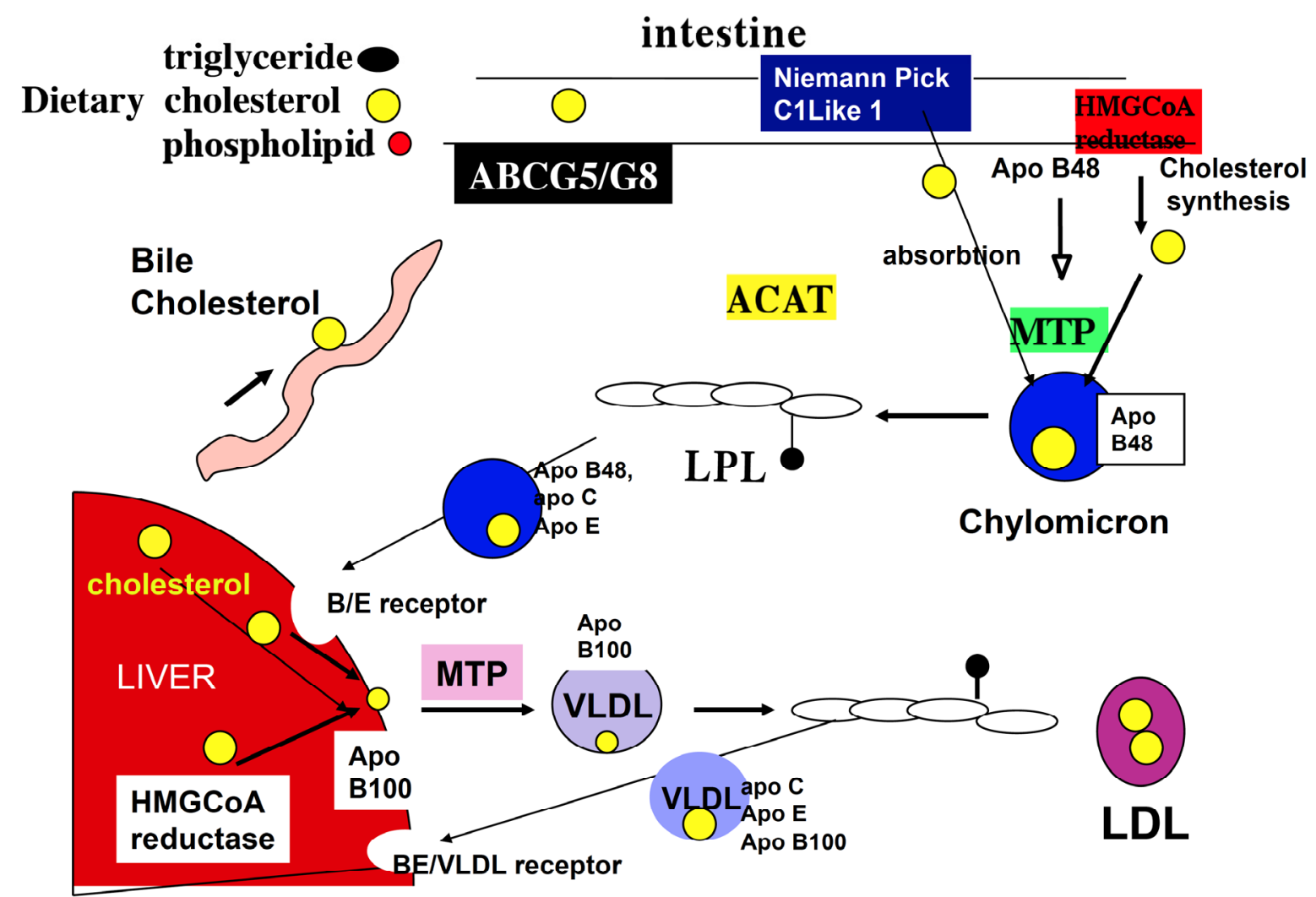

Fig. (1). Dietary cholesterol, biliary cholesterol and cholesterol synthesized in the intestine for which HMGCoA is the rate limiting enzyme, is transported across the cell membrane by NPC1L1 and, together with triglyceride, phospholipid, and the intestinally derived apoB48 protein, is assembled, under the influence of MTP into the triglyceride-rich chylomicron. Some of the absorbed cholesterol is excreted back into the lumen of the intestine under the influence of ABCG5/G8. The chylomicron is partially hydrolysed in the circulation by lipoprotein lipase and acquires apo CIII and apo E. The resulting chylomicron remnant is taken up by the B/E receptor in the liver. The cholesterol and triglyceride released are re-assembled with hepatically synthesised cholesterol and apo B100 to form VLDL. Lipoprotein lipase in the artery wall releases the triglyceride from VLDL and it acquires apo CIII and apo E. Some of the VLDL is taken up again by the liver and the rest is further hydrolised and looses apo CIII and E to become IDL and then LDL.

they are responsible for controlling cholesterol re-excretion into the bile [8-10].

\section{LDL COMPOSITION}

Patients in the coronary care unit frequently do not have raised LDL cholesterol but the quality of LDL may be altered. In 1995 Austin and Krauss [11] described the association between small dense LDL and atherosclerosis. The LDL particle is a cholesterol-rich, triglyceride-poor particle. LDL is composed of a hydrophilic surface layer of phospholipid, free cholesterol and hepatically-derived apo B100 to package the particle and add stability. The core of the particle includes esterified cholesterol and triglyceride together with the fatty acid tails of the phospholipid. As with most proteins in the circulation, the particle may act as a carrier for other insoluble particles such as free fatty acids which may be loosely attached [12]. Perhaps more importantly lipoprotein lipase also attaches to the particle and this facilitates attachment of the particle to the endothelial surface. We have previously shown that lipoprotein lipase was increased on diabetic LDL [12]. LDL can be sub-divided into sizes by gradient gel electrophoresis and separated into a pattern $\mathrm{A}$ and a pattern $\mathrm{B}$, pattern $\mathrm{B}$ being termed small dense LDL [13]. This pattern has been associated with an increase in atherosclerosis but it has been difficult to define changes in composition of the LDL that create the increased atherogenicity. The usual way to separate the different sizes of apo B containing lipoproteins is by ultracentrifugation but the correlation between the denser particles on ultracentrifugation and electrophoresis is uncertain. The most recent addition to the methods to investigate lipoproteins is magnetic resonance (MR) spectroscopy which can sort particle size in large numbers of samples over very short time but this technique still does not define small dense LDL [14]. Some years ago a subfraction of LDL with oxidized characteristics was described and was named electronegative LDL (LDL') based on its properties of electrical mobility [15] and later re-named minimally oxidized LDL. More heavily oxidized LDL is more electronegative than $\mathrm{LDL}^{-}$and is identified as $\mathrm{LDL}^{-}$. It now appears that electronegative LDL may also be produced by phospholipase A2. Rosenson et al., in the PLASMA11 Trial [16] have shown that an inhibitor of PLA2 reduced LDL by $7 \%$ and small dense LDL by $11 \%$. Enrichment of LDL with 
apo CIII contributes to the electronegativity [17]. Anti LDLmonoclonal antibody had a protective effect against atherosclerosis in LDL receptor knock-out mice [18]. It has been suggested that LDL- is a potential stress biomarker present in health and disease [19]. Small dense LDL isolation by various methods has been compared by Cheung [20]. The suggestion is that LDLs atherogenicity resides in its large amount of cholesterol being packaged in a relatively small volume hence the surface area of the particle is relatively large making it more easily amenable to modification and therefore more avidly taken up by scavenger receptors. Small dense LDL is also more susceptible to glycation even in non-diabetic people [21]. The association between small dense LDL and VLDL has been investigated, not least because of the difficulty of demonstrating hypertriglyceridaemia as an independent risk factor for atherosclerosis. VLDL, like LDL comes in many sizes depending on its triglyceride load and the Scottish and Finnish groups $[22,23]$ many years ago demonstrated the relationship between large triglyceride-rich VLDL and small dense LDL.

The reason why small dense LDL is associated with atherogenic risk has been debated for many years and familial hypercholesterolaemia, the cause of early atherosclerosis is associated with larger more buoyant LDL rather than small dense LDL [24, 25]. It has been proposed that the increasing surface area of the particle makes it more amenable to oxidation and glycation, both modifications being associated with antibody formation. The result is an increase in scavenger receptor/fc receptor uptake by the macrophage. Oxidation of the LDL particle depends on oxidation of the protein and/or fatty acids. Polyunsaturated but not monounsaturated fatty acids are amenable to oxidation hence a particle rich in linoleic acid is more susceptible to oxidation than one rich in oleic acid. An increase of free radical production occurs in hyperglycaemia of diabetes, one reason for the increased LDL oxidation that occurs in poorly controlled diabetes. The increase in free fatty acids in poorly controlled diabetes is associated with an increase in fatty acids attached to the LDL particle, a further potential cause for increased oxidation of the particle [12, 20] (Fig. 2).

\section{LIPOPROTEIN (A)}

$\mathrm{Lp}(\mathrm{a})$ is a variant of LDL that is covalently linked to apo B. Concentrations vary widely through different populations and more than $90 \%$ of this variant is determined by inherited DNA sequence variation [26]. Evidence that $L p(a)$ is a cause rather than a consequence of $\mathrm{CAD}$ has been strengthened by a report by Robert Clarke et al., [27] who found that two $\mathrm{Lp}$ (a) SNPs explain $36 \%$ of the variation in $\mathrm{Lp}(\mathrm{a})$ in their population. Both SNPs were associated with coronary disease and, after adjustment for the plasma $\mathrm{Lp}(\mathrm{a})$ lipoprotein level, the association between LPA genotypes and CAD are abolished. The mechanism whereby Lp(a) may be particularly atherogenic is through its binding and transportation of phospholipids [28]. However, due to the particle homology with plasminogen a pro-enzyme related to fibrinolysis $[29,30]$. It is suggested that the particle also plays a part in thrombosis. Combining a pro-atherogenic factor with an antifibrinolytic factor makes $L p(a)$ an interesting candidate for a link between plaque and stenosis and a likely risk factor for thrombotic events, including atherosclerotic occlusion [31]. More recently $\mathrm{Lp}$ (a) has been shown to interfere with Annexin A5 binding to the pro coagulant phosphatidyl serine. Annexin 5 is involved in anticoagulation on the endothelial surface and thus another mechanism whereby $\mathrm{Lp}$ (a) might promote thrombosis [32].

\section{HIGH DENSITY LIPOPROTEIN (HDL)}

There is a strong inverse correlation between triglycerides and HDL which has been known for many years. Low HDL is known to be an independent and powerful predictor of atherosclerosis and the functions of HDL have been

\section{Modified LDL}

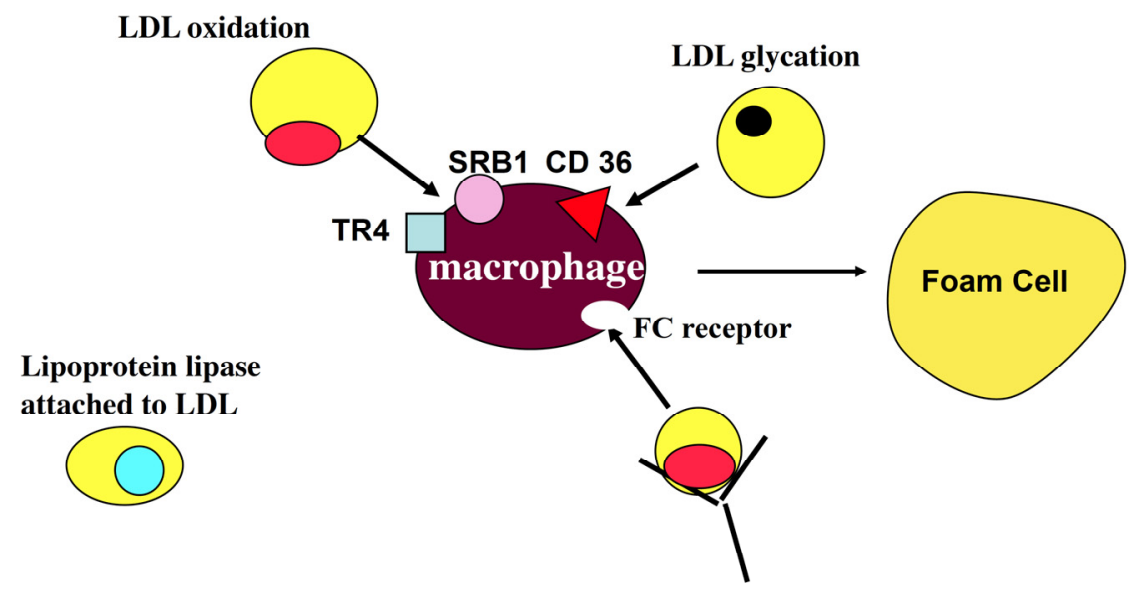

Oxidised LDL antibodies

Fig. (2). LDL may be modified by oxidation or glycation, and it may have increased lipoprotein lipase attached. Oxidised and glycated LDL are taken up by the macrophage scavenger receptors such as SRB1, CD36 and TR 4. Oxidised LDL may also form oxidized LDL-antibody complexes which can be taken up by the macrophage FC receptor. Scavenger receptor uptake is not regulated and leads to macrophage cholesterol accumulation and foam cell formation. 
intensively investigated. HDL's major function may be reverse cholesterol transport but it would seem that an important function relates to its ability of Paraoxinase 1 (PON 1) to protect LDL from oxidation. Thus, low HDL and, perhaps more importantly dysfunctional HDL such as is found in polymorphisms of PON 1, have a bearing on the atherogenicity of LDL [33].

\section{CAUSES OF HYPERCHOLESTEROLAEMIA}

Having discussed alterations in the particle that promote atherogenesis there is very good evidence to demonstrate a close relationship between the amount of cholesterol and the risk for example, of myocardial infarction. It is difficult to know how best to summarise the numerous studies and difficult to know which studies to select for discussion in this review. In the UK two fairly recent studies are worth mentioning. The Heart Protection Study [34] in which 20,536 people were non diabetic and 5,000 were diabetic and the CARDS Study [35] where all the 2838 patients were diabetic. These studies and many other studies have shown the benefits of reduction in cholesterol on cardiovascular end points. Although most patients with high levels of LDL cholesterol do not have a secondary cause such as hypothyroidism or nephrotic syndrome, dietary intervention leads to only a very moderate lowering of cholesterol i.e. in the Whitehall Study [36] in which 4469 patients completed the study, the patients who improved diet and lifestyle lowered cholesterol by only about $0.9 \mathrm{mmol} / 1$ over 9 years compared with $2.7 \mathrm{mmol} / 1$ for lipid lowering drug treatment. High LDL is often genetic in origin and, as with most common conditions there probably are multiple genes involved in the majority of patients. There are however rare monogenic causes of high LDL cholesterol [37].

Familial hypercholesterolaemia $(\mathrm{FH})$ is caused by a defect in the LDL receptor gene. The story of the discovery of the LDL receptor has been written by Goldstein and
Brown [1]. The story starts with Karl Muller [38] who described hypercholesterolaemia as an inborn error of metabolism and Goldstein the Nobel prize, came about by examination of homozygous patients. Their research led to the conclusion that a high affinity receptor existed with a feed back mechanism to suppress cholesterol synthesis through suppression of $\mathrm{HMGCoA}$ reductase. It is now known that this mechanism is mediated through sterol regulated binding protein (SREBP) [1]. Patients with $\mathrm{FH}$ lacked the high affinity receptor activity. Many different mutations of the LDL receptor gene have now been found in $\mathrm{FH}$ patients. LDL receptor gene transfer as a possible cure for FH is discussed in the recent review by Van Craeyveld et al., [39] (Fig. 3).

Proprotein convertase subtilisin kexin type 9 (PCSK9) is the gene which regulates the breakdown of the receptor [40]. PCSK9 is a regulator of liver LDL receptor expression. It binds tightly to the LDL receptor and channels it towards the lysosomal compartment for degradation. This results in decreased LDL receptor numbers and increased plasma LDL levels. An interesting loss of function polymorphism of PCSK9 increases the number of LDL receptors and increases LDL removal from the plasma, reducing LDL levels. There is strong evidence that PCSK9 and LDL receptor transcription are both activated by cellular cholesterol depletion via sterol regulatory element binding protein-2 (SREBP-2) [41]. This notion is supported by human studies where plasma PCSK9 concentration is increased with statin [42]. Noguchi et al., [43] have recently shown that fibrates also significantly increase circulating PCSK9 levels. Thus, an inhibitor of PCSK9 would be a useful addition to statin and fibrate therapy and Chan et al., [44] have recently described dramatic LDL lowering in non human primates with the use of monoclonal antibodies (mAB) against PCSK9 (Fig. 4).

\section{Regulated cellular uptake of LDL}

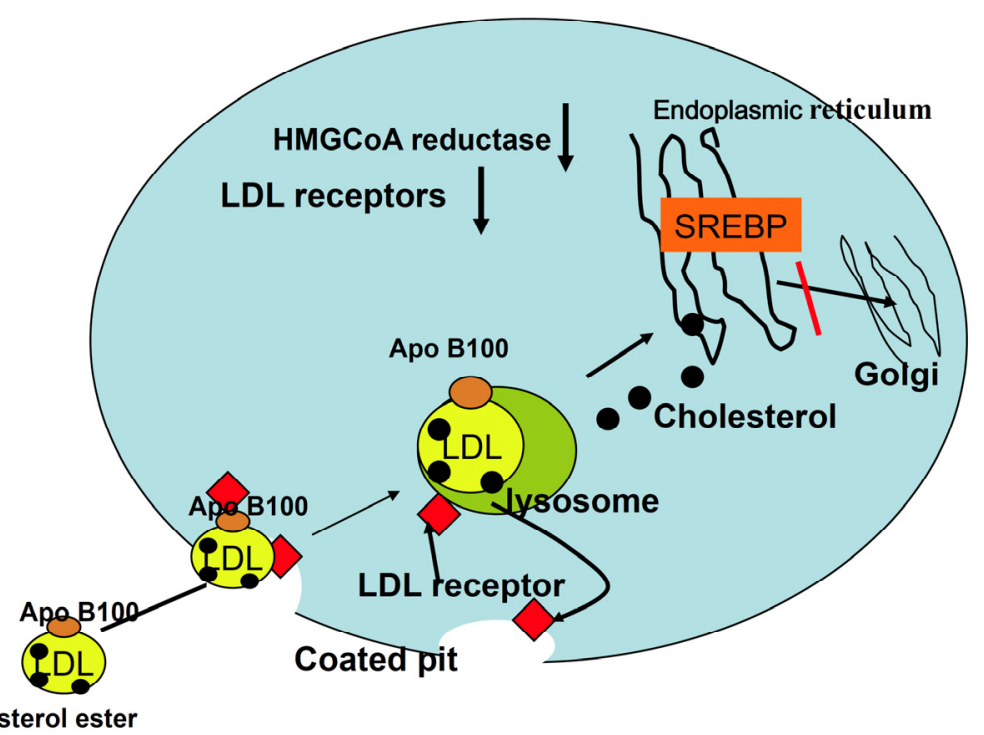

Fig. (3). Cholesterol taken into the cell through the LDL receptor pathway is released in the lysosome and taken up by the endoplasmic reticulum. This blocks the transport of SREBP to the Golgi complex preventing transcription of HMGCoA reductase thus reducing de novo cholesterol synthesis and also blocking LDL receptor synthesis, preventing further LDL uptake. 


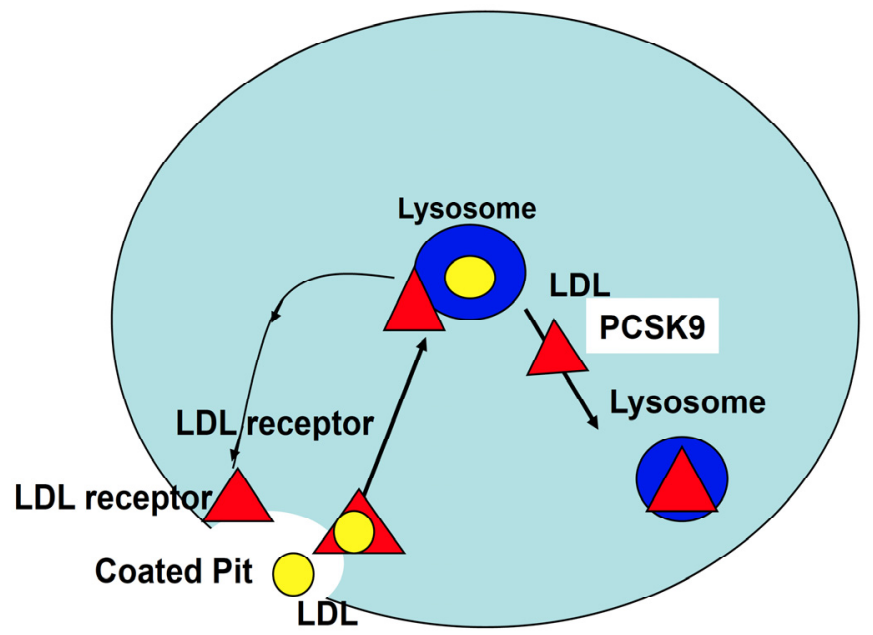

Fig. (4). PCSK9 is a regulator of liver LDL receptor expression. Normally the LDL receptor, once it has delivered LDL to the lysosome re-circulates to the coated pit on the cell surface. PCSK9 binds tightly to the LDL receptor and instead channels it towards the lysosomal compartment for degradation resulting in decreased LDL receptor numbers and increased plasma LDL cholesterol.

Apo B gene abnormalities which lead to abnormal apo B which is unable to bind effectively to the receptor is another monogenic cause of dyslipidaemia. These genetic defects can lead to either hyper- or hypocholesterolaemia. Mis-sense mutations in the LDL receptor domain of Apo B are characterised by premature atherosclerosis and coronary artery disease. Familial defective apolipoprotein B-100 (FDB) is a dominantly inherited disorder caused by the substitution of glutamine for arginine at position 3500 in apo B-100 [45]. The presence of mutant apo B-100 in lowdensity lipoproteins (LDL) markedly reduces its affinity for the LDL receptor, leading to hypercholesterolaemia and increased proneness to coronary artery disease. Other mutations in apo B can cause hypobetalipoprotinaemia characterised by hypocholesterolaemia and resistance to atherosclerosis. Here the defect in apo B results in defective cholesterol binding and LDL formation. [46- 49]

The genome-wide search looking for DNA variants that might impact on risk of coronary artery disease noted a DNA variant on chromosome $1 \mathrm{p} 13$ that increased the relative risk of coronary artery disease by $29 \%$ per allele [50]. The story as related in "clinical implications of basic research" $[51,52]$ describes the next milestone which was another genomewide association which found the above genetic variant was related to increased serum levels of LDL cholesterol [53]. In 2010 Musunuru et al., [54] discovered that the genes on $1 \mathrm{p} 13$ were associated with a transcription factor which led them to SORT-1 encoding sortilin 1 protein. Over expression of SORT1 in mice led to an increase in LDL cholesterol and knock down of SORT1 had the opposite effect explaining why the DNA variant on 1 p13 might increase the risk of coronary artery disease. It appears that sortilin 1 decreases particles containing apo $\mathrm{B}$ in the liver and over expression increases these particles. Even more interesting, the over expression of sortilin was shown to enhance the endocytosis of LDL particles in vitro $[51,52]$. Reduction of sortlin 1 expression regulates both VLDL and LDL and, as LinselNitschke [52] suggest, perhaps these new findings could ultimately translate into new approaches to treatment.
Autosomal recessive hypercholesterolaemia (ARH) is caused by another protein involved in transport of cholesterol from LDL via the LDL receptor. Cells from patients with $A R H$ fail to internalise the LDL receptor because they carry two defective alleles of LDL RAP1, a gene that encodes a specific clathrin adaptor protein [55]. Polymorphisms of the gene are rare but are associated with hypercholesterolaemia [56].

\section{FREE FATTY ACIDS}

In diabetes free fatty acid (FFA) suppression is impaired. It used to be generally believed that serum albumin was the protein carrier for these fatty acids although in 1995 Chung et al., [57] demonstrated that when hypertriglyceridaemic sera were lipolysed more than $80 \%$ of the FFAs were partitioned to the lipoprotein fractions, saturated FFAs having a 4.5 - 11 times greater partitioning into lipoproteins than into the albumin fraction relative to that of polyunsaturated FFAs. In vitro experiments by the authors showed that FFAs bound to LDL were highly cytotoxic to macrophages, whereas FFA partitioned to albumin were not. We found a10-fold increase of FFA on LDL from diabetic patients compared to controls [12]. We have demonstrated increased oxidisablity of LDL in both hypercholesterolaemic and normocholesterolaemic diabetic patients [58]. Patients with diabetes and metabolic syndrome have both a preponderance of small dense LDL and the increase in the polyunsaturated fatty acids in the particle, hence many important mechanisms that promote the atherogenicity of LDL in hyperglycaemia $[59,60]$.

\section{ATHEROSCLEROSIS FORMATION}

The mechanism by which LDL attaches itself to damaged intima in the arterial tree is a fascinating story. LDL carries not only apo B but other proteins including lipoprotein lipase. Lipoprotein lipase (LPL) is synthesised in tissue parenchymal cells and then translocated to functional binding sites on the luminal surfaces of endothelial cells. Heparin sulphate proteoglycans, located in lipid rafts in the endothelial cell surfaces and small capillaries are the docking stations for LPL [61]. Although LPL is mostly involved in lipolysis of triglycerides from triglyceride-rich lipoproteins, they are also important in LDL metabolism [61]. Proteoglycans are important players in the attachment of LDL particles to the vascular wall and act as a docking mechanism for the LDL particles (Fig. 5). We have shown an increase in LPL attached to LDL in diabetes [12] which may be important in the attachment of the LDL particle to the proteoglycans on the endothelial surface [62]. Antibodies to glycose aminoglycans have recently been shown to have an anti-atherogenic effect [63]. Platelet derived growth factor (PDGF) is strongly implicated in atherosclerosis, in part perhaps because it stimulates proteoglycan synthesis. For proof of concept Ballinger ML et al., [64] inhibited PDGF receptor. They demonstrated significant reduction of carotid artery lipid accumulation. The mechanism was through inhibiting glycosaminoglycan (GAG) synthesis on the proteoglycans and thus reducing LDL binding, a possible novel method for reducing atherosclerosis in the future. It has been demonstrated that hypoxia induces changes in macrophage GAG. GAG biosynthesis which lead to higher 


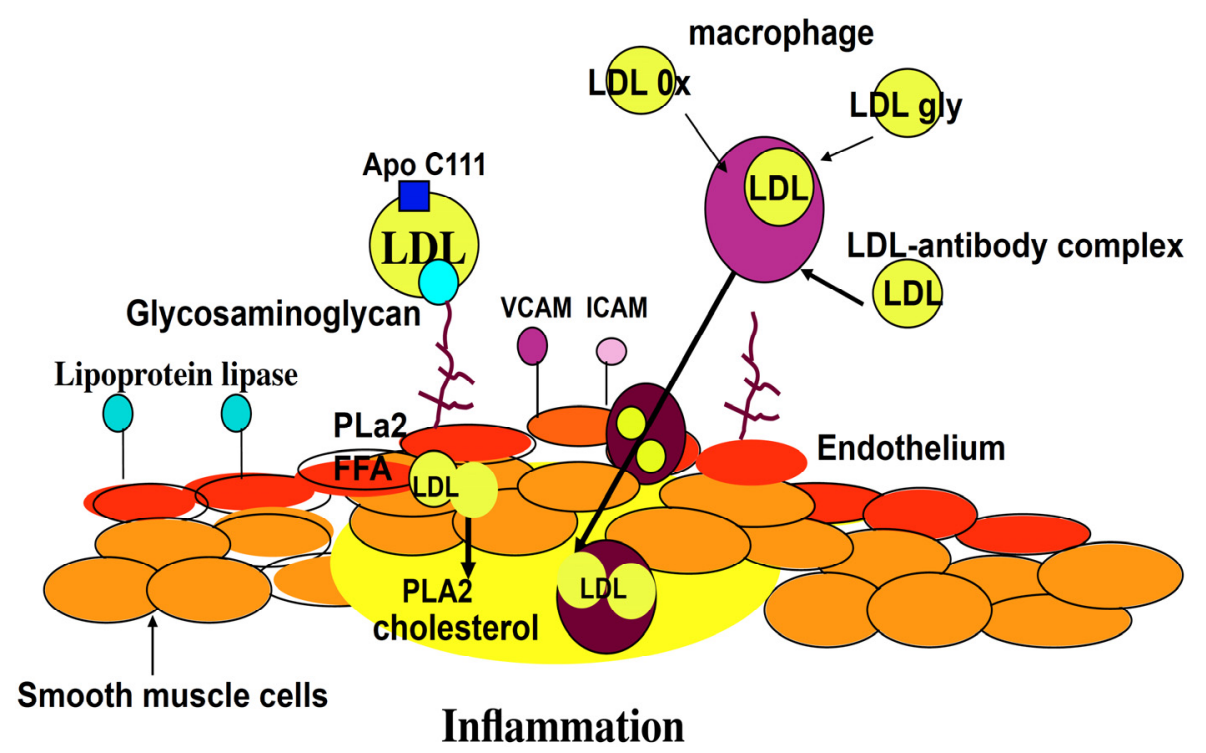

Fig. (5). The atherosclerotic plaque is composed of a lipid-rich core containing cholesterol, fatty acids and necrotic tissue, and is covered by a fibrous smooth muscle cell cap. Low density lipoprotein (LDL) is the major contributor to plaque cholesterol. LDL may attach to the endothelium through lipoprotein lipase and heparin sulphate proteoglycans (HPSG) which facilitate their uptake into the sub endothelial space. Macrophages which have accumulated large amounts of cholesterol, attach to chemotactic factors such as VCAM and ICAM on the artery wall, and slip between the endothelial cells into the intima where they are trapped, mature into foam cells and eventually disintegrate providing the cholesterol for the lipid-rich atherosclerotic core.

affinity for LDL and might contribute to the development of atherosclerosis.

Once the LDL particle has been attached to the endothelial surface further changes must occur before the cholesterol-rich particle becomes part of the atherosclerotic plaque. LDL aggregation seems very important in this process. The mechanisms whereby LDL particles fuse and coalesce into lipid droplets and thereby increase LDL in the artery wall is complex [65]. The LDL particle contains phospholipid. Phospholipase A2 (PLA2) is associated with atherosclerosis (Fig. 5). The effects of PLA2 and its products on structural stability of human LDL has been examined recently. Jayaraman et al., [66] demonstrated, in very elegant studies, that free fatty acids enhance LDL coalescence into lipid droplets. They promote the idea that lipid droplet formation contributes to the pro-atherogenic effects of FFA on LDL [66]. Most of the lipids found in atherosclerotic plaques are present in lipid droplets and LDL derived small lipid droplets are prominent in atherosclerotic lesions [67]. Modifications such as oxidation, lipolysis and proteolysis are prerequisites for droplet fusion. Enzymes from the Phospolipase A2 (PLA2) family hydrolyse phosphatidyl choline. Lipoprotein associated PLA2 preferentially hydrolyses oxidised phosphotidyl cholines in LDL and may promote fusion of LDL particles and thus contribute to the enhanced atherogenicity of the [68, 69]. Lipoprotein associated Phospholipase A2 has emerged as a causative agent of atherosclerosis and as a new therapeutic target. For review see [66]. It is suggested that the proatherogenic properties of PLA2 result from the effect of its products lysoPC and FFA, on LDL fusion and rupture, the released FFA promoting lipoprotein rupture and coalescence into lipid droplets. Interest in PLA2 has been heightened since inhibitors of PLA2 have been developed which demonstrate reduction in LDL concentration and in particular small LDL concentration suggesting that this class of drug may become an effective anti atherosclerotic agent [70].

The initial lesion leading to atherosclerosis is the fatty streak. Indeed fatty streaks occurs in human fetal aortas and are enhanced by maternal hypercholesterolaemia [71]. Analysis of the streak shows deposition of cholesterol and lipid with disruption of the endothelial surface and infiltration of macrophages and also neutrophils, typical of an inflammatory reaction. What is so interesting is that these streaks appear to repair themselves and do not lead to lasting damage. Delivery of cholesterol to the atherosclerotic plaque occurs following binding of the LDL particle to the endothelial surface via the lipase receptors. Endothelial dysfunction as measured by its failure to respond to nitric oxide, may be the first abnormality in the atherosclerotic process. Endothelial function impairment occurs before structural changes such as intimal hyperplasia or lipid deposition occur [72]. Endothelial dysfunction is associated with a decrease in nitric oxide production in the vasculature. Studies in the human coronary circulation have demonstrated that endothelial dysfunction is characterised by local enhancement of oxidative stress without a decrease in basal nitric oxide release [72]. Lavi et al., [73] suggest that their study supports the hypothesis that local oxidative stress has a role in reduction of NO bioavailability in humans with coronary endothelial dysfunction but the repair mechanism involves monocyte/ macrophage accumulation in an effort to clear cholesterol from the plaque. The macrophage does not have an LDL receptor and can only take up modified LDL. Modifications include oxidation, glycation and antibody formation which can occur in the sub endothelial space (Fig. 2). Once the macrophage takes up the LDL and becomes a foam cell it should by rites be allowed to remove cholesterol to the reticuloendothelial system for clearance but in the atherosclerotic process the macrophage gets trapped in the 
intima and is unable to escape due to increase in size. From the above simplistic description it is apparent that the LDL species depends for its atherogenicity to a large extent on its ability to be modified. There are numerous reviews of the oxidative process but in essence the release of free radicals is enhanced from conditions such as hyperglycaemia, ischaemia, infection and would be available to oxidise the particle [74]. The major sites of oxidation include apo B and the polyunsaturated fatty acids but cholesterol and phospholipids can also be oxidised. There is one apo B molecule /particle hence apo B is considered a strong risk marker for atherosclerosis. The amount of oxidised phospholipid on LDL apo B100 appears to be a good marker of atherosclerotic progression [75].

Apo CIII is a strong risk factor for cardiovascular disease. Apo CIII increases the binding of LDL to artery wall proteoglycans and increases accumulation of lipoproteins in the vascular wall $[76,77]$. Huikka et al., [78] have suggested that CIII-enriched LDL from diabetic patients might bind preferentially because of other changes in LDL since adding extra CIII to LDL did not increase binding to any great extent. The authors found that diabetic LDL with high endogenous CIII content contained less unesterified cholesterol and more triglyceride. They further found that the high apo CIII content in diabetic LDL was associated with increased esterified cholesterol, sphingomyelin, ceremide and the ceremide containing ganglioside GM1. They suggest that these changes may be associated with higher membrane fluidity and higher freedom in lateral movement thus allowing Apo B to acquire a conformation which is more favorable for proteoglycan binding. The heparin sulphate proteoglycans (HSPG) play an important role in the assembly and structure of the basement membrane [79]. There is evidence that HSPG is reduced under hyperglycaemic conditions [80]. Loss of arterial heparin sulphate correlates with the onset of atherosclerosis in a monkey model of diabetes [81]. Recently, Rao et al., [79] have shown that hyperglycaemia induces heparin sulphate degradation through increase reactive oxygen production via increase in heparanase 1 which specifically degrades heparin sulphate proteoglycans thus a vicious circle is set up in which an increase in free radical production increases oxidation and Lp (a) aggregation and in turn stimulates inflammatory molecules such as tumor necrosis factor- $\alpha(\mathrm{TNF} \alpha)$ and interleukan6 (IL-6) and increase free radical production. Heparanase- 1 is produced locally by the endothelium. TNF $\alpha$, interleukin $1 \beta$ (IL-1 $\beta$ ) and fatty acids are able to induce heparanase-1 production in endothelial cells in vitro. Rao et al., [79] showed abundant heparanase-1 staining in endothelial cells from atherosclerotic plaques suggesting that many pathological factors in the atherosclerotic plaque can contribute to heparanase-1 production in endothelial cells. Thus increased heparanase-1 production in endothelial cells and macrophages may be responsible for decreased amounts of heparin sulphate proteoglycans in the arterial wall and may contribute to the pathogenesis of atherosclerosis.

The foam cell is perhaps the hallmark of the atherosclerotic lesion. The development of the foam cell depends on macrophage uptake of cholesterol. A novel mechanism has recently been described. The Toll-like receptor 4 (TR4) recognises minimally oxidised LDL [82,
83] (Fig. 2). Choi et al., [84] demonstrated a complex signalling pathway induced by minimally oxidised LDL led to enhanced uptake of small molecules such as minimally oxidised LDL, resulting in lipid accumulation. Thus the authors have described a novel mechanism leading to enhanced LDL uptake in macrophages that would lead to foam cell formation and atherosclerosis. In diabetes we and others have shown an increase in LDL esterified cholesterol so it is interesting that Choi et al., [84] demonstrated that cholesterol ester hydroperoxides are an indigenous ligand for TR4.

\section{THROMBUS FORMATION}

Thrombus formation on atherosclerotic lesions is a late stage in the disease and leads to myocardial infarction and stroke. Tissue factor [85] is a potent pro-coagulant and matrix degrading proteinase that is involved in the rupture of the fibrous cap and promotes thrombosis. Macrophages are major source within the atherosclerotic plaque of tissue factor, a membrane bound glycoprotein receptor that triggers the thrombotic cascade. Recently Sardo et al., [86] have demonstrated that low concentrations of oxidised LDL enhance tissue factor expression but higher concentrations attenuate tissue factor expression. Perhaps another reason why minimally oxidised LDL is potentially so atherogenic. Oxidised phospholipids derived from modified lipoproteins and in particular LDL, stimulate monocyte expression of urokinase and urokinase receptors which promote the secretion of matrix metalloproteinase-9 (MMP-9). Cellular exposure to MMP-9 also promotes nuclear factor $\mathrm{kb}$ expression in the mononuclear cell, another factor involved in destabilisation of plaque and thrombus formation $[87,88]$.

\section{CONCLUSION}

The evidence is overwhelming that elevated and/or modified LDL is a major risk factor for atherosclerosis. The atherosclerotic potential depends, not only on mass but also on composition. In addition HDL has a major impact on the atherogenicity of LDL. The interrelationship between all lipoprotein particles is so strong that preventative treatment should not focus on LDL alone but should encompass the whole lipoprotein family. Basic research has identified new targets for treatment that have the potential to reverse atherosclerosis to a much greater extent than is possible at present.

\section{ACKNOWLEDGEMENT OF FUNDING}

Diabetes Research Foundation Dublin Ireland.

\section{CONFLICT OF INTEREST}

None declared.

\section{REFERENCES}

[1] Goldstein JL, Brown MS. The LDL receptor. Atherioscler Thromb Vasc Biol 2009; 29: 431-8.

[2] 4S Investigators. Randomised trial of cholesterol lowering in 4444 patients with coronary heart disease: the Scandinavian Simvastatin Survival Study (4S) Lancet 1994 ; 344: 1383-9.

[3] Taylor F, Ward K, Moore TH, et al. Statins for the primary prevention of cardiovascular disease. Cochrane Database Syst Rev 2011(1): CD 004816.

[4] Khera AV, Cuchel M, de la Llera-Moya M, et al. Cholesterol efflux capacity, high-density lipoprotein function, and atherosclerosis. N Engl J Med 2011; 364: 127-35. 
[5] Brown MS, Dana SE, Goldstein JL. Regulation of 3-hydroxy-3methylglutaryl coenzyme A reductase activity in cultured human fibroblasts: comparison of cells from a normal subject and from a patient with homozygous familial hypercholesterolemia. J Biol Chem 1974; 249: 789-96.

[6] Tremblay AJ, Lamarche B, Lemelin V, et al. Atorvastatin increases intestinal expression of NPC1L1 in hyperlipidemic men. J Lipid Res 2011; 52: 558-65.

[7] Altmann SW, Davis HR Jr, Zhu IJ, et al. Niemann- Pick C1 Like 1 protein is critical for intestinal cholesterol absorption. Science 2004; 303: 1201-4.

[8] Berge KE, Tian H, Graf GA, et al. Accumulation of dietary cholesterol in sitosterolemia caused by mutations in adjacent $\mathrm{ABC}$ transporters. Science 2000; 290: 1771-5.

[9] Graf GA, Yu L, Li WP, et al. ABCG5 and ABCG8 are obligate heterodimers for protein trafficking and biliary excretion. J Biol Chem 2003; 278: 48275-82.

[10] Kosters A, Kunne C, Looije N, Patel SB, Oude Elferink RP, Groen AK. The mechanism of ABCG5/ABCG8 in biliary cholesterol secretion in mice. J Lipid Res 2006; 47: 1959-66.

[11] Austin MA, Kraus RM. LDL density and atherosclerosis. JAMA 1995; 373: 115.

[12] Phillips C, Owens D, Mullan K, Tomkin GH. Low density lipoprotein non-esterified fatty acids and lipoprotein lipase in diabetes. Atherosclerosis 2005; 181: 100-14.

[13] Krauss RM, Burke DJ. Identifiation of multiple subclasses of plasma low density lipoprotein in normal humans. J lipid Res 1982; 23: 97-104.

[14] Blake GJ, Otvos JD, Rifai N, Ridker PM. Low-density lipoprotein particle concentration and size as determined by nuclear magnetic resonance spectroscopy as predictors of cardiovascular disease in women. Circulation 2002; 106: 1930-7.

[15] Avogaro P, Bon GB, Cazzolato G. Presence of a modified low density lipoprotein in humans. Arteriosclerosis 1988; 8: 79-87.

[16] Rosenson RS, Elliott M, Stasiv Y, Hislop C. PLASMA II Investigators. Randomized trial of inhibitor of secretory phospholipase A2 on atherogenic lipoprotein subclasses in statin- treated patients with coronary heart disease. Eur Heart J 2011; 32: 999-1005.

[17] Hiukka A, Ståhlman M, Pettersson C. Apo CIII enriched LDL in type 2 diabetes displays altered lipid composition, increased susceptibility for sphingomyelinase, and increased binding to biglycan. Diabetes 2009; 58: $2018-26$

[18] Grosso DM, Ferderbar S, Wanschel AC, Krieger MH, Higushi ML, Abdalla DS. Antibodies against electronegative LDL inhibit atherosclerosis in LDLr-/- mice. Braz J Med Biol Res 2008; 41: 108692.

[19] Mello AP, da Silva IT, Abdalla DS, Damasceno NR. Electronegative low-density lipoprotein: origin and impact on health and disease. Atherosclerosis 2011; 215: 257-65.

[20] Chung M, Lichtenstein AH, Ip S, Lau J, Balk EM. Comparability of methods for LDL subfraction determination: a systematic review. Atherosclerosis 2009; 20: 342-8.

[21] Younis N, Charlton MV, Sharma R, Soran H, Durrington PN. Glycation of LDL in non- diabetic people: small dense LDL is preferentially glycated both in vivo and in vitro. Atherosclerosis 2009; 202: 162-8.

[22] Taskinen MR. Diabetic dyslipidaemia: from basic research to clinical practice. Diabetologia. 2003; 46: 733-49.

[23] Gaw A, Packard CJ, Lindsay GM, et al. Overproduction of small very low density lipoproteins (Sf 20-60) in moderate hypercholesterolemia: relationships between apolipoprotein B kinetics and plasma lipoproteins. J Lipid Res 1995; 36: 158-71.

[24] Mikhailidis DP, Elisaf M, Rizzo M, et al. "European Panel on Low Density Lipoprotein (LDL) Subclasses": a statement on the pathophysiology, atherogenicity and clinical significance of LDL subclasses. Curr Vasc Pharmacol 2011; 9: 533-71.

[25] Mikhailidis DP, Elisaf M, Rizzo M, et al. "European Panel on Low Density Lipoprotein (LDL) Subclasses": a statement on the pathophysiology, atherogenicity and clinical significance of LDL subclasses: executive summary. Curr Vasc Pharmacol 2011; 9: 531-2.

[26] Kathiresan $\mathrm{S}$. $\mathrm{Lp}$ (a) lipoprotein redux--from curious molecule to causal risk factor. N Engl J Med 2009; 361: 2573-4.

[27] Clarke R, Peden JF, Hopewell JC, et al. PROCARDIS Consortium Genetic variants associated with $\mathrm{Lp}(\mathrm{a})$ lipoprotein level and coronary disease. N Engl J Med 2009; 361: 2518- 28.
[28] Enkhmaa B, Anuurad E, Zhang W, Tran T, Berglund L. Lipoprotein (a): genotype- phenotype relationship and impact on atherogenic risk. Metab Syndr Relat Disord 2011; 9: 411-8.

[29] Koschinsky ML. Lipoprotein (a) and atherosclerosis: new perspectives on the mechanism of action of an enigmatic lipoprotein. Curr Atheroscler Rep 2005; 7: 389-95.

[30] Koschinsky ML. Lipoprotein (a) and the link between atherosclerosis and thrombosis. Can J Cardiol 2004; 20 (Suppl B): 37B-43B.

[31] Berglund L, Ramakrishnan R. Lipoprotein (a): an elusive cardiovascular risk factor. Arterioscler Thromb Vasc Biol 2004; 24: 2219-26.

[32] Fu YC, Yang JT, Chen HW, Wu JH. Effect of lipoprotein (a) on annexin A5 binding to cell membrane. Clin Chim Acta. 2010; 411: 1915-9.

[33] Kontush A, Chapman MJ. Antiatherogenic function of HDL particle subpopulations: focus on antioxidative activities. Curr Opin Lipidol 2010; $21: 312-8$.

[34] Heart Protection Study Collaborative Group. MRC/BHF Heart Protection Study of cholesterol lowering with simvastatin in 20,536 high-risk individuals: a randomised placebo- controlled trial. Lancet 2002; 360: 7-22.

[35] Colhoun HM, Betteridge DJ, Durrington PN, et al. CARDS investigators; primary prevention of cardiovascular disease with atorvastatin in type 2 diabetes in the Collaborative Atorvastatin Diabetes Study (CARDS): multicentre randomised placebo-controlled trial. Lancet 2004; 364: 685-96.

[36] Bouillon K, Singh MA, Jokela M, et al. Decline in low-density lipoprotein cholesterol concentration: lipid-lowering drugs, diet, or physical activity? Evidence from the Whitehall II Study. Heart 2011; 97: 923-30.

[37] Goldstein JL, Brown MS. Molecular medicine. The cholesterol quartet. Science 2001; 292: 1310-2.

[38] Muller C. Xanthomata, hypercholesterolaemia, angina Pectoris. Acta Med Scand 1938; 89: 75-84.

[39] Van CE, Jacobs F, Gordts SC, De Geest B. Gene therapy for familial hypercholesterolemia. Curr Pharm Des 2011; 17: 2575-91.

[40] Abifadel M, Varret M, Rabès JP, et al. Mutations in PCSK9 cause autosomal dominant hypercholesterolemia. Nat Genet 2003; 34: 154-6.

[41] Jeong HJ, Lee HS, Kim KS, Kim YK, Yoon D, Park SW. Steroldependent regulation of proprotein convertase subtilisin/kexin type 9 expression by sterol-regulatory element binding protein-2. J Lipid Res 2008; 49: 399-409.

[42] Careskey HE, Davis RA, Alborn WE, Troutt JS, Cao G, Konrad RJ. Atorvastatin increases human serum levels of proprotein convertase subtilisin/kexin type 9. J Lipid Res 2008; 49: 394-8.

[43] Noguchi T, Kobayashi J, Yagi K, et al. Comparison of the effects of bezafibrate and fenofibrate on circulating proprotein convertase subtilisin/kexin type 9 and adipocytokine levels in dyslipidemic subjects with impaired glucose tolerance or type 2 diabetes mellitus: results from a crossover study. Atherosclerosis 2011;217: 165-70.

[44] Chan JC, Piper DE, Cao Q, et al. A proprotein convertase subtilisin/kexin type 9 neutralizing antibody reduces serum cholesterol in mice and nonhuman primates. Proc Natl Acad Sci USA 2009; 106: 9820-5.

[45] Miserez AR, Keller U. Differences in phenotypic characteristics of subjects with familial defective B100 and familial hypercholesterolaemia Atherosclerosis. Thromb Vasc Biol 1995; 15; 1719-29.

[46] Myant NB. Familial defective apo B100; a review including some comparisons with hypercholesterolaemia. Atherosclerosis 1993; 104: 118.

[47] Vega GL, Grundy SM. In vivo evidence for reduced binding of low density lipoproteins to receptors as a cause of primary moderate hypercholesterolemia. J Clin Invest 1986; 78: 1410-14.

[48] Soria LF, Ludwig EH, Clarke HR, Vega GL, Grundy SM, McCarthy BJ. Association between a specific apolipoprotein B mutation and familial defective apolipoprotein B-100. Proc Natl Acad Sci USA 1989; 86: 587-91.

[49] Innerarity TL, Mahley RW, Weisgraber KH, et al. Familial defective apolipoprotein B-100: a mutation of apolipoprotein B that causes hypercholesterolemia. J Lipid Res 1990; 31: 1337-49.

[50] Samani NJ, Erdmann J, Hall AS, et al. Cardiogenics Consortium, Genomewide association analysis of coronary artery disease. N Engl J Med 2007; 357: 443-53.

[51] Linsel NP, Samani NJ, Schunkert H. Sorting out cholesterol and coronary artery disease. N Engl J Med 2010; 363: 2462-3. 
[52] Linsel NP, Schunkert H. Chromosome 9p21 and coronary risk--the mystery continues. Atherosclerosis 2011;214: 257-8.

[53] Kathiresan S, Willer CJ, Peloso GM, et al. Common variants at 30 loci contribute to polygenic dyslipidemia. Nat Genet 2009; 41: 56-65.

[54] Musunuru K, Post WS, Herzog W, et al. Association of single nucleotide polymorphisms on chromosome 9p21.3 with platelet reactivity: a potential mechanism for increased vascular disease. Circ Cardiovasc Genet 2010; 3: 445-53.

[55] Garcia CK, Wilund K, Arca M, et al. Autosomal recessive hypercholesterolemia caused by mutations in a putative LDL receptor adaptor protein. Science 2001; 292: 1394-8.

[56] Soutar AK. Rare genetic causes of autosomal dominant or recessive hypercholesterolaemia. IUBMB Life 2010; 62: 125-31.

[57] Chung BH, Tallis GA, Cho BH, Segrest JP, Henkin Y. Lipolysisinduced partitioning of free fatty acids to lipoproteins: effect on the biological properties of free fatty acids. J Lipid Res 1995; 36: 1956-70.

[58] Bowie A, Owens D, Collins P, Johnson A, Tomkin GH. Glycosylated low density lipoprotein is more sensitive to oxidation: implications for the diabetic patient? Atherosclerosis 1993; 102: 63-7.

[59] Sugino I, Kuboki K, Matsumoto T, Murakami E, Nishimura C, Yoshino G. Influence of fatty liver on plasma small, dense LDLcholesterol in subjects with and without metabolic syndrome. J Atheroscler Thromb 2011; 18: 1-7.

[60] Scheffer PG, Tushuizen ME, Vermue HP, Schindhelm RK, Rustemeijer C, Diamant M. Effect of three consecutive meals on the physicochemical properties of HDL and LDL in individuals with the metabolic syndrome and patients with type 2 diabetes. Eur J Clin Nutr 2011; 165(11): 1242-9. doi: 10.1038/ejen.2011.114.

[61] Wang H, Eckel RH. Lipoprotein lipase: from gene to obesity. Am J Physiol Endocrinol Metab 2009; 297: E271-88.

[62] Braun JEA, Severson DL. Regulation of the synthesis, processing and translocation of lipoprotein lipase. Biochem J 1992; 287: 337-47.

[63] Soto Y, Acosta E, Delgado L, et al. Antiatheroslerotic effect of an antibody that binds to extracellular matrix glycosaminoglycans. Arterioscler Thromb Vasc Biol 2012; 32(3): 595-604.

[64] Ballinger ML, Osman N, Hashimura K, et al. Imatinib inhibits vascular smooth muscle proteoglycan synthesis and reduces LDL binding in vitro and aortic lipid deposition in vivo. J Cell Mol Med 2010; 14: 140818.

[65] Oörni K, Pentikäinen MO, Ala-Korpela M, Kovanen PT. Aggregation, fusion, and vesicle formation of modified low density lipoprotein particles: molecular mechanisms and effects on matrix interactions. J Lipid Res 2000; 41: 1703-14.

[66] Jayaraman S, Gantz DL, Gursky O. Effects of phospholipase A2 and its products on structural stability of human LDL: relevance to formation of LDL-derived lipid droplets. J Lipid Res 2011; 52: 549-57.

[67] Asplund A, Fridén V, Stillemark BP, Camejo G, Bondjers G. Macrophages exposed to hypoxia secrete proteoglycans for which LDL has higher affinity. Atherosclerosis 2011; 215: 77-81.

[68] Guyton JR, Klemp KF. Development of the atherosclerotic core region., chemical and ultrastructural analysis of microdissected atherosclerotic lesions from human aorta. Arterioscler Thromb 1994; 14: 1305-14

[69] Bancells C, Benítez S, Ordóñez-Llanos J, et al. Immunochemical analysis of the electronegative LDL subfraction shows that abnormal Nterminal apolipoprotein B conformation is involved in increased binding to proteoglycans. J Biol Chem 2011; 14: 1125-33.

[70] Rosenson RS, Elliott M, Stasiv Y. Hislop C for the PLASMA II Investigators, randomized trial of an inhibitor of secretory phospholipase $\mathrm{A} 2$ on atherogenic lipoprotein subclasses in statin-treated patients with coronary heart disease. Eur Heart J 2011; 8: 999-1006.

[71] Napoli C, D'Armiento FP, Mancini FP, et al. Fatty streak formation occurs in human fetal aortas and is greatly enhanced by maternal hypercholesterolemia, intimal accumulation of low density lipoprotein and its oxidation precede monocyte recruitment into early atherosclerotic lesions. J Clin Invest 1997; 100: 2680-90.

[72] Napoli C, Ignarro LJ. Nitric oxide and pathogenic mechanisms involved in the development of vascular diseases. Arch Pharm Res 2009; 32: 1103-8.

[73] Lavi S, Yang EH, Prasad A, et al. The interaction between coronary endothelial dysfunction, local oxidative stress, and endogenous nitric oxide in humans. Hypertension 2008; 51: 127-33.

[74] Steinberg D, Witztum JL. Oxidized low-density lipoprotein and atherosclerosis. Arterioscler Thromb Vasc Biol 2010; 30: 2311-6.

[75] Ahmadi N, Tsimikas S, Hajsadeghi F, et al. Relation of oxidative biomarkers, vascular dysfunction, and progression of coronary artery calcium. Am J Cardiol 2010; 105: 459-66.

[76] Davidsson P, Hulthe J, Fagerberg B, et al. Proteomic study of the apolipoproteins in LDL subclasses in patients with the metabolic syndrome and type 2 diabetes. J Lipid Res 2005; 46: 1999- 2006.

[77] Olin LK, Krauss RM, La Belle M, et al. ApoC-III content of apoBcontaining lipoproteins is associated with binding to the vascular proteoglycan biglycan. J Lipid Res 2002; 43: 1969-77.

[78] Hiukka A, Ståhlman M, Pettersson C, et al. ApoCIII- enriched LDL in type 2 diabetes displays altered lipid composition, increased susceptibility for sphingomyelinase, and increased binding to biglycan. Diabetes 2009; 58: 2018-26.

[79] Rao G, Ding HG, Huang W, et al. Reactive oxygen species mediate high glucose- induced heparanase-1 production and heparan sulphate proteoglycan degradation in human and rat endothelial cells: a potential role in the pathogenesis of atherosclerosis. Diabetologia 2011; 54: 1527-38.

[80] Wasty F, Alavi MZ, Moore S. Distribution of glycosaminoglycans in the intima of human aortas: changes in atherosclerosis and diabetes mellitus. Diabetologia 1993; 36: 316-22.

[81] Edwards IJ, Wagner JD, Vogl-Willis CA, Litwak KN, Cefalu WT. Arterial heparan sulfate is negatively associated with hyperglycemia and atherosclerosis in diabetic monkeys. Cardiovasc Diabetol 2004; 29: 3-6.

[82] Miller YI, Viriyakosol S, Binder CJ, Feramisco JR, Kirkland TN, Witztum JL. Minimally modified LDL binds to CD14, induces macrophage spreading via TLR4/MD-2, and inhibits phagocytosis of apoptotic cells. J Biol Chem 2003; 278: 1561-8.

[83] Miller YI, Viriyakosol S, Worrall DS, Boullier A, Butler S, Witztum $\mathrm{JL}$. Toll-like receptor 4- dependent and independent cytokine secretion induced by minimally oxidized low density lipoprotein in macrophages. Arterioscler Thromb Vasc Biol 2005; 25: 1213-9.

[84] Choi SH, Harkewicz R, Lee JH, et al. Lipoprotein accumulation in macrophages via toll-like receptor-4-dependent fluid phase uptake. Circ Res 2009; 104: 1355-63.

[85] Hasenstab D, Lea H, Hart CE, Lok S, Clowes AW. Tissue factor over expression in rat arterial neointimal models thrombosis and progression of advanced atherosclerosis. Circulation 2000; 101: 2651-7.

[86] Sardo MA, Campo S, Mandraffino G, et al. Tissue factor and monocyte chemoattractant protein-1 expression in hypertensive individuals with or without increased carotid intima-media wall thickness. Clin Chem 2008; 54: 814-23.

[87] Bruni F, Puccetti L, Pasqui AL, et al. Differential effect induced by treatment with several statins on monocyte tissue factor expression in hypercholesterolemic subjects. Clin Exp Med 2003; 3: 45-53.

[88] Nakagomi A, Sasaki M, Ishikawa Y, et al. Upregulation of monocyte tissue factor activity is significantly associated with carotid intima media thickness in patients with metabolic syndrome. J Atheroscler Thromb 2011; 18: 475-86. 